\title{
Remote Operated Unmanned Ground Vehicle
}

\author{
${ }^{1}$ Akshay D Kukanur \\ Student, Department of EEE, Tontadarya College of \\ Engineering, Gadag, Karnataka, India \\ ${ }^{2}$ Dada Khalandar \\ Student, Department of EEE, Tontadarya College of \\ Engineering, Gadag, Karnataka, India
}

\author{
${ }^{3}$ Maruthi V \\ Student, Department of EEE, Tontadarya College of \\ Engineering, Gadag, Karnataka, India \\ ${ }^{4}$ Malagoud C Patil \\ Student, Department of EEE, Tontadarya College of \\ Engineering, Gadag, Karnataka, India
}

\author{
${ }^{5}$ Jagadeeshwar G. Shivanagutti \\ Assistant Professor, Department of EEE, \\ Tontadarya College of Engineering, Gadag, \\ Karnataka, India
}

\begin{abstract}
Some of the major problems that our world is facing is terrorism, emergency evacuation operations and rising revolts. The human intervention in some cases may be hazardous to humans. The development of remote controlled unmanned ground vehicle assists humans in active combat and can collect the data from areas where human intervention is not possible.

Our UGV system has two parts one is the controlling unit of the UGV which tracks, monitors, controls and records the data from the UGV. The other part is the moving part which has various sensors and circuits that record and transmit the surrounding environmental changes to the control unit.
\end{abstract}

\section{Keywords-UGV-Unmanned Ground Vehicle;}

\section{INTRODUCTION}

The Remote Controlled Unmanned Ground vehicle has a various sensors and circuits placed on it. These sensors and circuits sense the changes in the surrounding and transmit the data to control station. These UGV receive commands from a distant operator through wired or wireless communication. Unlike the autonomous UGV which can self assist, remote controlled UGV's require command input from the human controller. The Camera and other vision Sensors can provide the live feed of the location. The humidity temperature sensors can provide the surrounding environment data. Proximity sensor used can detect any metal or land mines laid below the ground. The gps device can give the location of the vehicle. [2]

The use of UGV can help in gaining information in hazardous condition or where human intervention is not possible. These UGV have a wide variety of use. The military applications of the UGV can be for target locating, surveillance and recoinnassance. These UGV's have a great scope in space applications, agriculture, rescue and recovery operations, supply chain management etc., The foster milon talon swords are UGV's designed by the US army to assist soldiers in active combat. DRDO's DAKSH is another UGV designed by DRDO for detecting IED's and diffusing them.[4]
The objectives of UGV are:

\section{OBJECTIVES}

- The UGV's are built to undertake missions such as border patrol, reconnaissance and target acquisition.

- To assist in recovery and rescue operations .

- Surveillance in remote places.

- To collect and record environmental changes in inhabitable places.

- To detect landmines.

\section{SYSTEM ARCHITECTURE}

The mechanical design of the UGV demand for a robust design which makes the UGV operable in terrain and harsh environmental conditions. The chasis designed should be capable of holding the weight and pressure exerted on the UGV. The electrical design consists of different batteries and power supply, as different sensors and different components use different power supply. [2]

The UGV uses four dc motors for the movement of vehicle. The web IP camera at the front of the vehicle allows you to have the live video of the location using which you can steer the vehicle. The obstacle detection system designed using HC-SR 04 sensor helps in knowing the distance of the obstacle from the vehicle.[3]

The UGV consists of various parts. Each part performs a specific function. The various parts in the architecture are ;

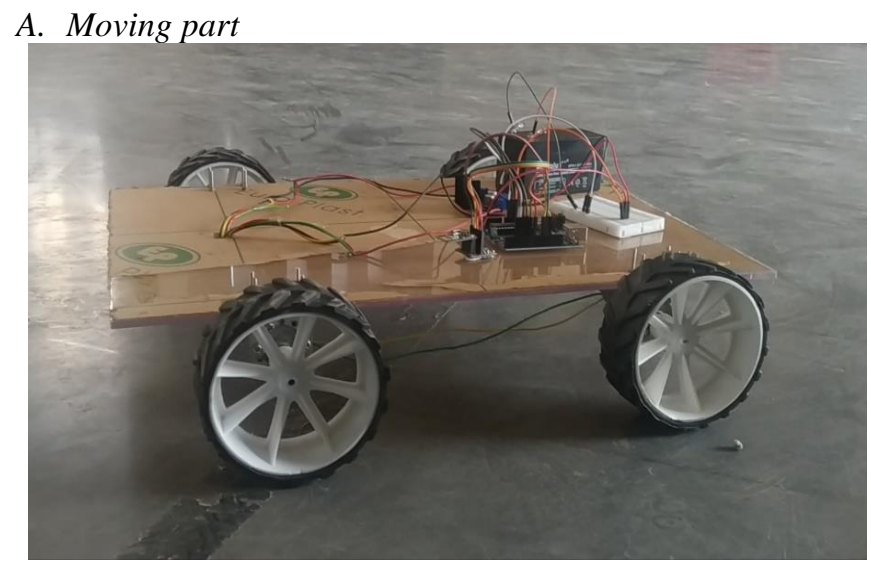

Fig 1(a) Moving part 
The moving part has an on board system on the UGV and an control unit.The onboard system is placed on the vehicle and consists of arduino microcontroller and motor drivers that allows the movement of the vehicle. The micro conntroller is connected to a NRF 24L01 radio module which receives signals from the control unit. The control unit has a joystick module connected to the arduino microcontroller, the NRF 24L01 module transmits the data from the joystick to the onboard system.[2]

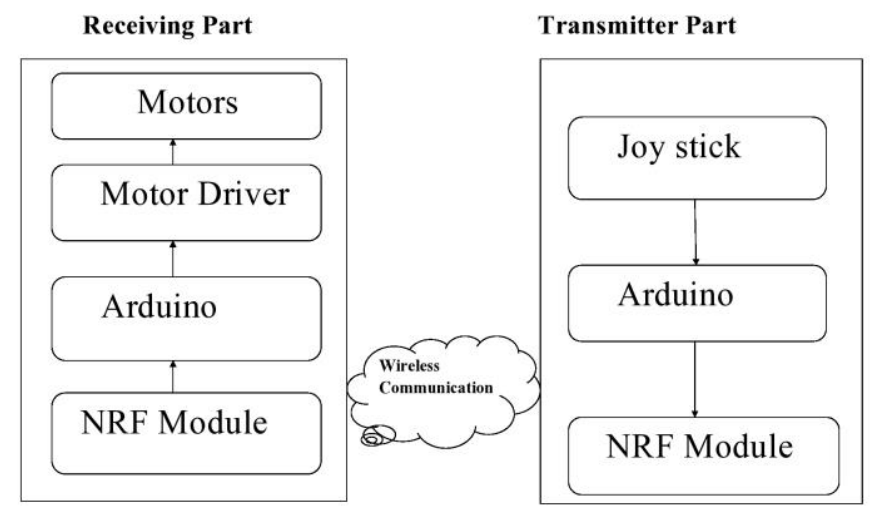

Fig 1(b) Block diagram of Moving part

\section{B. Surrounding Monitoring System}

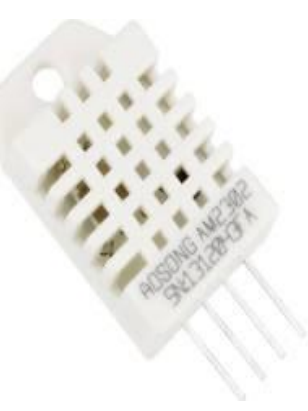

Fig 2(a) DHT22 sensor

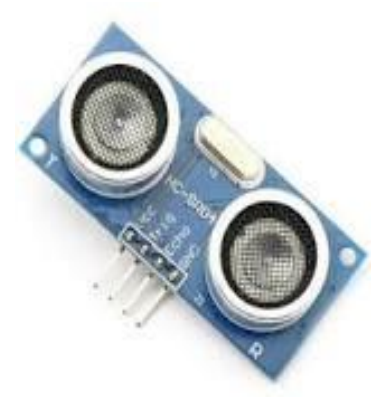

Fig 2(b) HC-SR 04
The surrounding monitoring system uses a DHT22 sensor to record the temperature and humidity of the surrounding. A HC-SR 04 sensor helps detecting obstacles in front of vehicle. The DHT22 and HC-SR 04 sensors are connected to an arduino. The arduino has a NRF 24L01 radio module connected which transmits the data recorded on the on board system. Control unit has a receiver part having a NRF24L01 module connected to arduino which receives data from on board system and is displayed on the computer.[1][3]

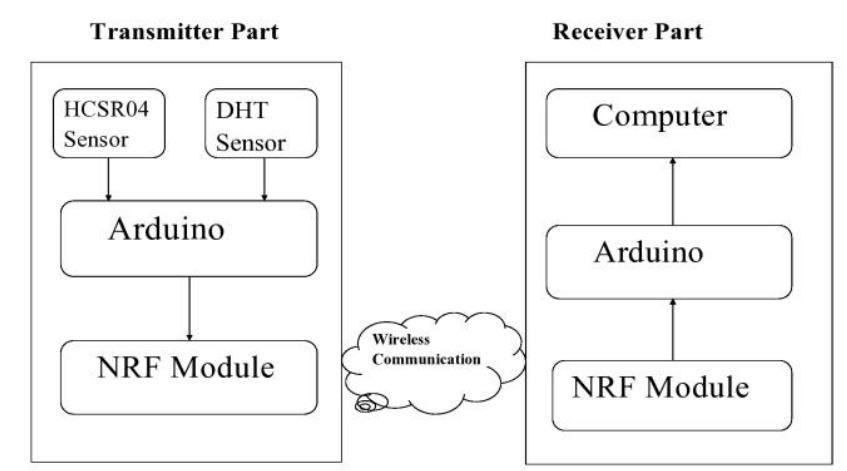

Fig 2(c) Block diagram of surrounding monitoring system C. Metal Detector

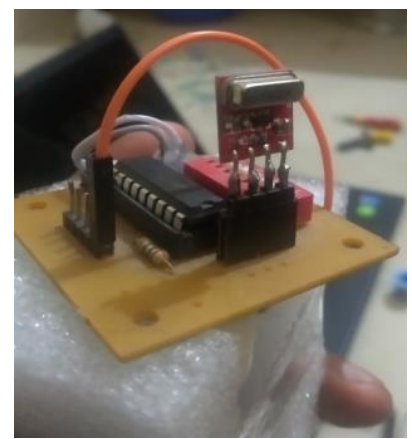

Fig 3(a) Metal detector circuit
Fig 3 (b) Proximity sensor

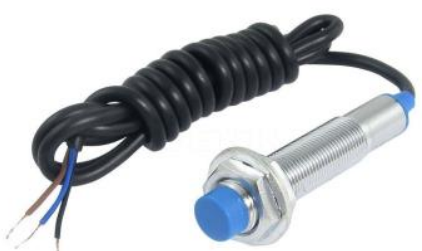

The metal detector uses a proximity sensor to detect any metal objects laid underground. The proximity sensor on the on board system is connected to a HT12E encoder which is capable of transfering 12 bit parallel data using an RF transmitter. The control unit has a receiver RF module connected to a HT12D decoder which is conneted to the arduino. Whenever a metal is detected the transmitter RF module transmits an active high signal. The receiver RF module receives the active high signal to the Decoder. The decoder decodes the signal and transmits data to computer through arduino.[1]

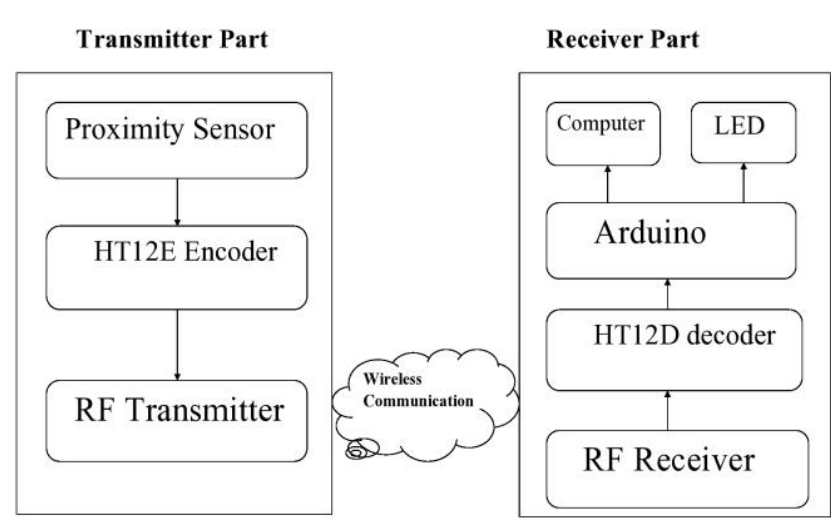

Fig 3(c) Block diagram of metal detector 
D. Tracking Device

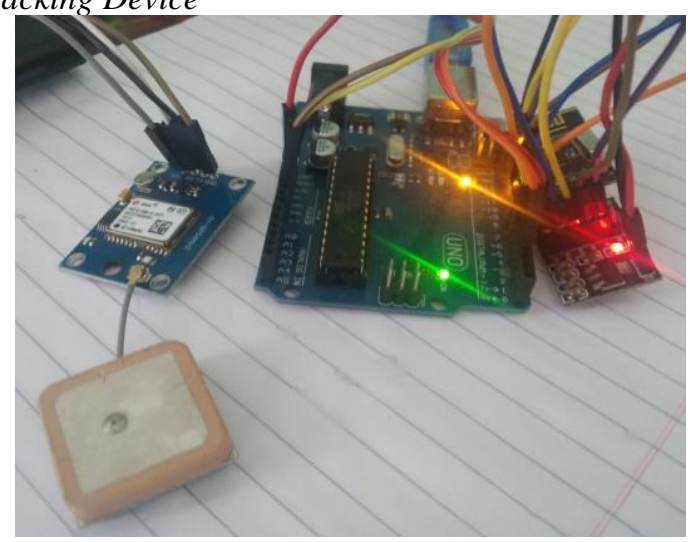

Fig 4(a) Tracking device

The tracking sytem of the vehicle uses a neo-6M GPS module.

GPS module is placed on the on board system and is connected to a arduino. The Latitude and longitude coordinates along with date and time are transmitted from the on board system using NRF module. The control unit has a NRF module connected to arduino which receives the coordinates, date and time from the on board system and displays it on the computer.[4]

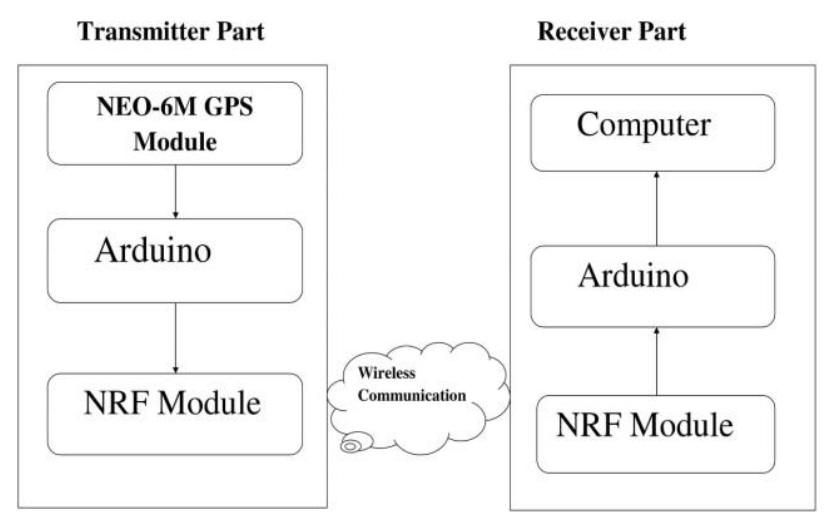

Fig 4 (b) Block diagram of tracking system

\section{E. Equations}

The surrounding monitoring system uses DHT sensor and a HC-SR 04 sensor. The DHT sensor monitors the temperature and humidity of the surrounding. The HCsr04 calculates the distance of the objects surrounding the vehicle. The HCsr04 sensor is ultrasonic sensor that sends the sound waves from transmitter, the sound waves hits the object and reflects back. The time taken by the wave to travel the distance is used to calculate the distance of the object.

The DHT sensor records the temperature and humidity of the surrounding and feeds to the microcontroller. The microcontroller uses the values of temperature and humidity to calculate the speed of sound waves in the surrounding.

The relation below gives the speed of the sound:

soundspeed $=331.4+(0.606 *$ temperature $)+(0.0124 *$ Humidity);
Using the sound speed the distance of the object from the UGV is calculated.

The formula for distance of the object is given by

Distance $=(\text { duration } / 2)^{*}$ sound speed in $\mathrm{cm}$

Duration $=$ Time taken by the sound waves to travel and detect the objects. Obtained by sonar iterations.

\section{ADVANTAGES AND APPLICATIONS}

A. Advantages

- Reduced manpower for surveillance purposes.

- Human intervention in hazardous conditions can be avoided.

- No need for humans to travel and record surrounding environment data.

- Reduce combat fatalities.

- Loss of lives caused by overstepping on land mines can be avoided.

\section{B. Application}

- Assist soldiers in active combat.

- Helps in Surveillance, reconnaissance and target acquisition.

- Space applications.

- Agricultural applications.

- Supply chain management.

- Help provide supplies to soldiers.

\section{CONCLUSION}

The reliability on humans in emergency situation is reduced, saving lives of soldiers and other servicemen. These UGV can assist and provide supplies to soldiers in active combat. The development in field of UGV's has resulted in making lives easier and reducing risks. The human intervention in hazardous condition can be avoided to much greater extent and manpower used can be reduced.

\section{ACKNOWLEDGEMENT}

The satisfaction of the successful completion of any task would be incomplete without the expression of gratitude to the people who have made it possible. We acknowledge all the people who have guided and encouraged us. We would like to take this opportunity to thank our guide Prof. Jagadeeshwar G. Shivanagutti, Assistant Professor, Department of Electrical and Electronics Engineering, for his immense guidance and support. We express our deep sense of gratitude to our beloved H.O.D Dr. Iranna. M. Korachagaon. Vice Principal and Head of Department of Electrical and Electronics Engineering, for his kind support. We extend our gratitude to Dr. M. M. Awati Principal, Tontadarya College of Engineering, Gadag, for his generous support in all regards.

\section{REFERENCES}

[1] Obstacle Detecting System of Unmanned Ground Vehicle Hee Chang Moon' , Hong Chul Lee2 and Jung Ha Kim3Graduate School of Automotive Engineering, Kookmin University, Seoul, Korea

[2] Design and Implementation of Low Cost Remote-Operated Unmanned Ground Vehicle , Zain Murtaza, Noaman Mehmood, Mohsin Jamil, 
Yasar Ayaz School of Mechanical and Manufacturing Engineering Department of Robotics \& AI National University of Sciences and Technology Islamabad, Pakistan

[3] Unmanned Ground Vehicle Using Ultrasonic Ranging module.Krutika Basinge, Sejal Nagpure, Punam Shinde, Prof.Y.K.Sharma VIIT , Pune.

[4] Saurav Kumar and Pallavi Awasthi, "Navigation Architecture for Autonomous Surveillance Rover," International Journal of Computer Theory and Engineering, Vol. 1, No. 3, August, 2009 1793-8201, Pg. 231-235..

\section{AUTHOR DETAILS}

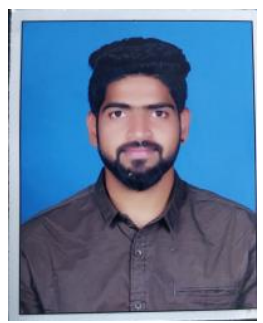

${ }^{1}$ Akshay D kukanur was born in Mundaragi Town, Gadag District, Karnataka State, India, on $14^{\text {th }}$ October 1996. He is now pursuing in B.E $\left(4^{\text {th }}\right.$ year) in Electrical and Electronics Engineering, Tontadarya College of Engineering, Gadag, Karnataka affiliated to Visvesvaraya Technological University, Belagavi, Karnataka. He is interested in power system and Power Electronics.

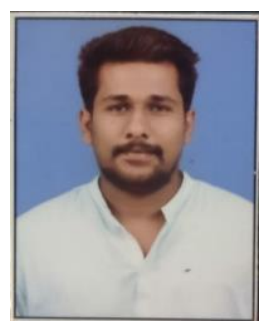

${ }^{2}$ Dada Khalandar was born in Koppal District, Karnataka State, India, on $28^{\text {th }}$ February 1996. He is now pursuing in B.E $\left(4^{\text {th }}\right.$ year $)$ in Electrical and Electronics Engineering, Tontadarya College of Engineering, Gadag, Karnataka affiliated to Visvesvaraya Technological University, Belagavi, Karnataka. $\mathrm{He}$ is interested in Transmission Distribution system, computer engineering and Renewable Energy.

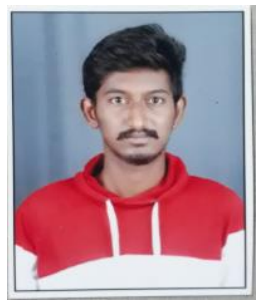

${ }^{3}$ Maruthi V was born in Hosadurga Town, Chitradurga District, Karnataka State, India, on $20^{\text {th }}$ January, 1997. He is now pursuing in B.E ( $4^{\text {th }}$ year) in Electrical and Electronics Engineering, Tontadarya College of Engineering, Gadag, Karnataka affiliated to Visvesvaraya Technological University, Belagavi, Karnataka. $\mathrm{He}$ is interested in Renewable Energy Sources, Transmission and Distribution System .

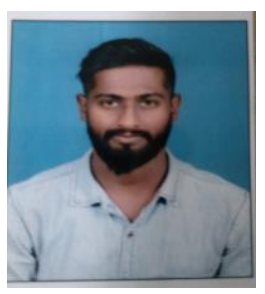

${ }^{4}$ Malagoud C Patil was born in Mantur Village, Raibag Town, Belgaum District, Karnataka State, India, on $22^{\text {nd }}$ November, 1995. He is now pursuing in B.E $\left(4^{\text {th }}\right.$ year $)$ in Electrical and Electronics Engineering, Tontadarya College of Engineering, Gadag, Karnataka affiliated to Visvesvaraya Technological University, Belagavi, Karnataka. $\mathrm{He}$ is interested in Electrical Power Generation and Transmission Distribution System.

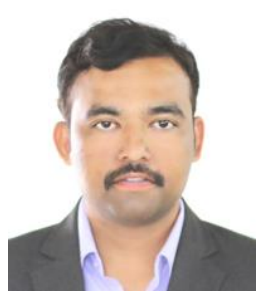

${ }^{5}$ Jagadeeshwar. G. Shivanagutti was born on 26th June, 1986 in Kushtagi, Koppal District, Karnataka State, India. $\mathrm{He}$ completed his Engineering Graduation from PDACE, Gulbarga, in Electrical Stream. Then he completed his M.Tech in Energy Systems and Management from SJCE, Mysore. Currently He is working as Assistant Professor in Tontadarya College of Engineering(NBA accredited), Gadag, Karnataka. He has been guiding several UG Projects. His areas of interests include Energy Conservation, Solar Energy Harnessing, Estimation and Utilization, Energy Management, Energy Audit and Renewable Energy Sources, Electrical Vehicles. He has published many international and national papers. His membership includes International Association of Engineers (IAENG), Institute, IAENG Society of Industrial Engineering \& IAENG Society of Electrical Engineering, Life time Professional Member of the Institute of Scholars. Life time professional member of Institute For Engineering Research and Publication (IFERP). He is a nominated faculty under NISP Implementation Program in HEI by MHRD Innovation Cell, Government of India. He received "InSc Best Teacher Award 2019" from Institute of Scholars(InSc). 